\title{
Age-driven developmental drift in the pathogenesis of idiopathic pulmonary fibrosis
}

\author{
Moisés Selman ${ }^{1}$, Carlos López-Otín² and Annie Pardo ${ }^{3}$
}

\author{
Affiliations: \\ ${ }^{1}$ Instituto Nacional de Enfermedades Respiratorias Ismael Cosío Villegas, Mexico city, Mexico. \\ ${ }^{2}$ Departamento de Bioquímica y Biología Molecular, Facultad de Medicina, Instituto Universitario de \\ Oncología, Universidad de Oviedo, Oviedo, Spain. \\ ${ }^{3}$ Facultad de Ciencias, Universidad Nacional Autónoma de México, Mexico city, Mexico.
}

\section{Correspondence:}

Moisés Selman, Instituto Nacional de Enfermedades Respiratorias, Tlalpan 4502, CP 14080, México DF, México.

E-mail: mselmanldyahoo.com.mx

ABSTRACT Idiopathic pulmonary fibrosis (IPF) is a progressive and usually lethal disease of unknown aetiology. A growing body of evidence supports that IPF represents an epithelial-driven process characterised by aberrant epithelial cell behaviour, fibroblast/myofibroblast activation and excessive accumulation of extracellular matrix with the subsequent destruction of the lung architecture. The mechanisms involved in the abnormal hyper-activation of the epithelium are unclear, but we propose that recapitulation of pathways and processes critical to embryological development associated with a tissue specific age-related stochastic epigenetic drift may be implicated. These pathways may also contribute to the distinctive behaviour of IPF fibroblasts. Genomic and epigenomic studies have revealed that wingless/ Int, sonic hedgehog and other developmental signalling pathways are reactivated and deregulated in IPF. Moreover, some of these pathways cross-talk with transforming growth factor- $\beta$ activating a profibrotic feedback loop. The expression pattern of microRNAs is also dysregulated in IPF and exhibits a similar expression profile to embryonic lungs. In addition, senescence, a process usually associated with ageing, which occurs early in alveolar epithelial cells of IPF lungs, likely represents a conserved programmed developmental mechanism. Here, we review the major developmental pathways that get twisted in IPF, and discuss the connection with ageing and potential therapeutic approaches.

@ERSpublications

Twisted stochastic age-driven developmental drift and antagonistic pleiotropy contribute to aberrant repair in IPF http://ow.ly/LN5W300wHOj 


\begin{abstract}
Introduction
Idiopathic pulmonary fibrosis (IPF) is a chronic, progressive and usually lethal disorder of unknown aetiology and very limited therapeutic options [1]. The disease is characterised by the aberrant activation of epithelial cells, which secrete numerous mediators resulting in the expansion of the fibroblast/ myofibroblast population with the subsequent exaggerated accumulation of extracellular matrix and the destruction of the lung architecture [2]. IPF is the most common of the idiopathic interstitial pneumonias and the incidence and prevalence seems to be increasing over time. Confident diagnosis relies in the presence of the so-called usual interstitial pneumonia (UIP) pattern on high-resolution computed tomography and/or on surgical lung biopsy (figure 1).
\end{abstract}

Although the pathogenic mechanisms leading to IPF have not been elucidated, ageing seems to be a major driving force. Thus, patients are usually over 50 years old at the time of diagnosis, and the incidence increases remarkably beyond the fifth decade of life [1-4]. Most of the hallmarks that characterise the ageing process have been identified in IPF lungs including abnormal shortening of telomeres, genomic instability, mitochondrial dysfunction, increased oxidative stress and senescence, which affect primarily the alveolar epithelial cells [4-9]. In addition, several environmental and occupational exposures have been reported as risk factors, but smoking is the most consistently recognised risk factor for developing both the sporadic and the familial forms of IPF [1]. However, it is important to emphasise that accelerated lung ageing and smoking are also associated with other chronic, degenerative lung disorders such as chronic obstructive pulmonary disease (COPD) [10].

In this context, a growing body of evidence indicates a complex scenario for the development of IPF. We have proposed that the conjunction of a distinct genetic architecture (a set of gene variants affecting primarily the epithelial integrity), plus ageing-related biopathology and epigenetic reprogramming, results in abnormal epithelial cell activation and the initiation/development of IPF [5]. However, the mechanisms by which gene-environmental risk factors contribute to this epithelial cell activation have not been deciphered. Here we propose that an aberrant recapitulation of developmental pathways induced by a tissue-specific stochastic profibrotic age-related epigenetic drift plays a key role. In fact, the epigenome shows a significant drift with age. This is in part because of stochastic replication-dependent errors in the maintenance of epigenetic states (random ageing-epigenetic drift model), although there are also specific genomic regions that follow distinctive ageing patterns of DNA methylation.

\title{
Transcriptional signatures of IPF recapitulate embryological pathways
}

IPF lungs are significantly enriched with genes associated with relevant signalling pathways involved in lung development [11]. Moreover, the comparison of transcriptional signatures of IPF with other interstitial lung diseases has revealed that $\sim 20 \%$ of the dysregulated genes in IPF are related to development [12]. The upregulated development-related genes include several members of transcription factor families such as the Sry-related high mobility group box and forkhead box, and genes related to the $\mathrm{Wnt} / \beta$-catenin $(\mathrm{Wnt})$ and sonic hedgehog $(\mathrm{SHH})$ signalling pathways [11].

\section{Wnt and SHH signalling pathways are upregulated in IPF and cross-talk with transforming growth factor- $\beta$}

The Wnt family consists of 19 secreted glycoproteins that bind the frizzled receptor and subsequently recruit the downstream signal mediators dishevelled leading to the nuclear accumulation of $\beta$-catenin to
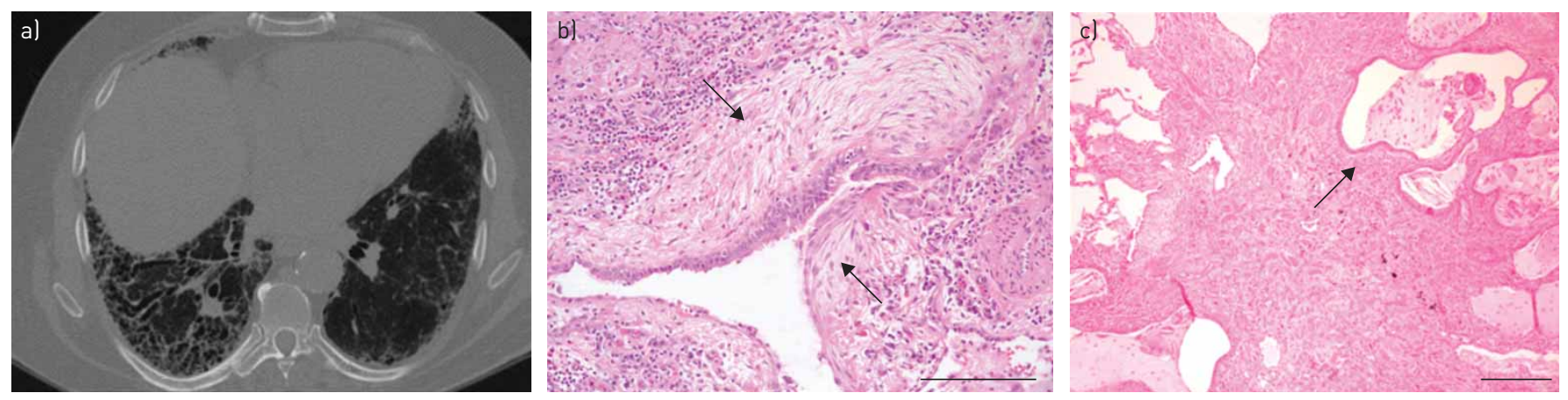

FIGURE 1 a) High-resolution computed tomography in a 73 year-old patient with idiopathic pulmonary fibrosis. Computed tomography shows changes consistent with the usual interstitial pneumonia pattern, characterised by reticular opacities, traction bronchiectasis, small ground-glass opacifications and honeycombing, manifested as subpleural, clustered cystic airspaces with well-defined walls. b and c) Histopathological features of usual interstitial pneumonia. b) Fibroblasts foci, representing small areas of active fibrosis, are present in the background of collagen deposition beneath an abnormal epithelium (arrows). Scale bar $=50 \mu \mathrm{m}$. c) Dense fibrosis and honeycomb lesions (arrow). Scale bar=500 $\mu \mathrm{m}$. 
initiate transcription of target genes. It was first demonstrated that IPF lungs exhibited nuclear accumulation of $\beta$-catenin in alveolar epithelial type 2 cells (AEC2) [13]. Then, several Wnt ligands as well as glycogen synthase kinase- $3 \beta$ and $\beta$-catenin were found to be highly expressed in hyperplastic AEC2 and bronchiolar epithelial cells indicating that the $\mathrm{Wnt} / \beta$-catenin signalling pathway is activated in the epithelium during IPF development $[14,15]$. In particular, Wnt1 inducible protein 1 (WISP1), a signalling molecule downstream of the Wnt signalling pathway, was highly upregulated in IPF lungs and localised to hyperplastic AEC2 cells at sites of bronchiolisation. WISP1 treatment of AEC2 resulted in increased proliferation, expression of profibrotic genes and epithelial-to-mesenchymal transition (EMT) [15]. Upregulation of some Wnt ligands has also been revealed in IPF fibroblasts. For example, strongly immunoreactive Wnt7B and Wnt10A are focally localised in fibroblastic foci $[16,17]$. Interestingly, IPF patients' positive for Wnt10A showed a decreased survival time, probably associated with acute exacerbations [17]. Also, the non-canonical Wnt5A is increased in IPF fibroblasts. Furthermore, stimulation of fibroblasts with Wnt5A increased proliferation and resistance to apoptosis suggesting that it may contribute to fibroblast expansion and survival [18].

Subsequent to these findings, it was demonstrated that $\beta$-catenin and transforming growth factor (TGF)- $\beta$-dependent signalling pathways cooperate in the AEC2 to induce the transcription of genes associated with EMT [19].

More recently, it was also reported that SHH signalling, which controls the growth and survival of the embryonic lung mesenchyme and is required for proper lung branching morphogenesis, is strongly upregulated in the AECs of IPF lungs, while the transcription factor GLI1 was observed in both fibroblasts and hyperplastic epithelium $[20,21]$. SHH may contribute to IPF pathogenesis by increasing the proliferation, migration, extracellular matrix production and survival of fibroblasts [21]. SHH also cross-talks with TGF- $\beta 1$, which modulates the expression of key components of this pathway in human lung fibroblasts while GLI-dependent transcription was required for the profibrotic effects of TGF- $\beta 1$ [20].

Although Wnt and $\mathrm{SHH}$ are the most studied developmental pathways so far, other proteins that participate in embryonic lung development are also reactivated in IPF. For example, grainyhead-like 2 (GRHL2), a novel transcription factor involved in epithelial morphogenesis, is abundantly expressed in lung epithelium during development and is detected in lung mesenchyme only at the early fetal stage [22]. In IPF lungs, GRHL2 is expressed in collapsing alveolar epithelium, together with a surprising ectopic expression in fibroblasts, rather similar to the expression pattern observed at human embryonic weeks 911 [22]. GRHL2 may bind the hTERT promoter and contribute to telomerase activity [23]. Whether this function participates in the pathogenesis of IPF is still uncertain, but it may explain the finding of increased telomerase activity and telomere length in IPF fibroblasts [24].

Wilms tumour-1 (WT1), a zinc finger transcription factor that regulates many functional properties of the developing mesothelium, is reactivated in IPF [25]. Pleural mesothelial cells (PMCs) expressing WT1 have been revealed in IPF lungs, but not in cystic fibrosis or COPD [26]. WT-1 regulates PMCs plasticity, and might contribute through mesothelial-to-mesenchymal transition to the expansion of the myofibroblast population. Supporting this notion, WT1-positive mesenchymal cells were recognised as a subset of lung-resident mesenchymal cells that progressively accumulate in the subpleural fibrotic lesions of IPF, which may at least partially explain the peculiar subpleural initiation of the disease [27]. Forkhead box F1 (FOXF1) is another important factor for embryological development that is overexpressed in a variety of cell types in IPF lungs [28]. FOXF1 is expressed almost exclusively in fetal mesenchyme, where it controls key morphogenic functions. Interestingly, FOXF1 repressed proliferation and collagen expression in normal lung fibroblasts, suggesting an antifibrotic role. However, its effects were blunted in IPF fibroblasts. More recently, fibroblast growth factor (FGF) 9 and FGF18, two mediators critical for lung development, were found in the remodelled alveolar epithelium close to the fibroblast foci in IPF lungs [29]. However, the effect of these factors on the pathogenesis of IPF is uncertain, since in normal and IPF fibroblasts FGF9 promotes survival and migration (that may enhance the progression of fibrosis), but inhibits myofibroblast differentiation and collagen expression [29]. Other FGFs that have important functions during branching morphogenesis of lung epithelium have also been found to be overexpressed in IPF lungs (FGF2) or dysregulated in IPF fibroblasts [30, 31].

These findings indicate that, in IPF lungs, primarily epithelial cells but also fibroblasts demonstrate persistent activation of several highly conserved signal transduction pathways involved in development (figure 2).

\section{Finely regulated developmental pathways are also recapitulated during normal} lung regeneration

Signalling pathways, morphogen gradients and transcription factors that control critical stages of lung development are often reactivated after birth in post-injury tissue regeneration. The classical example in 


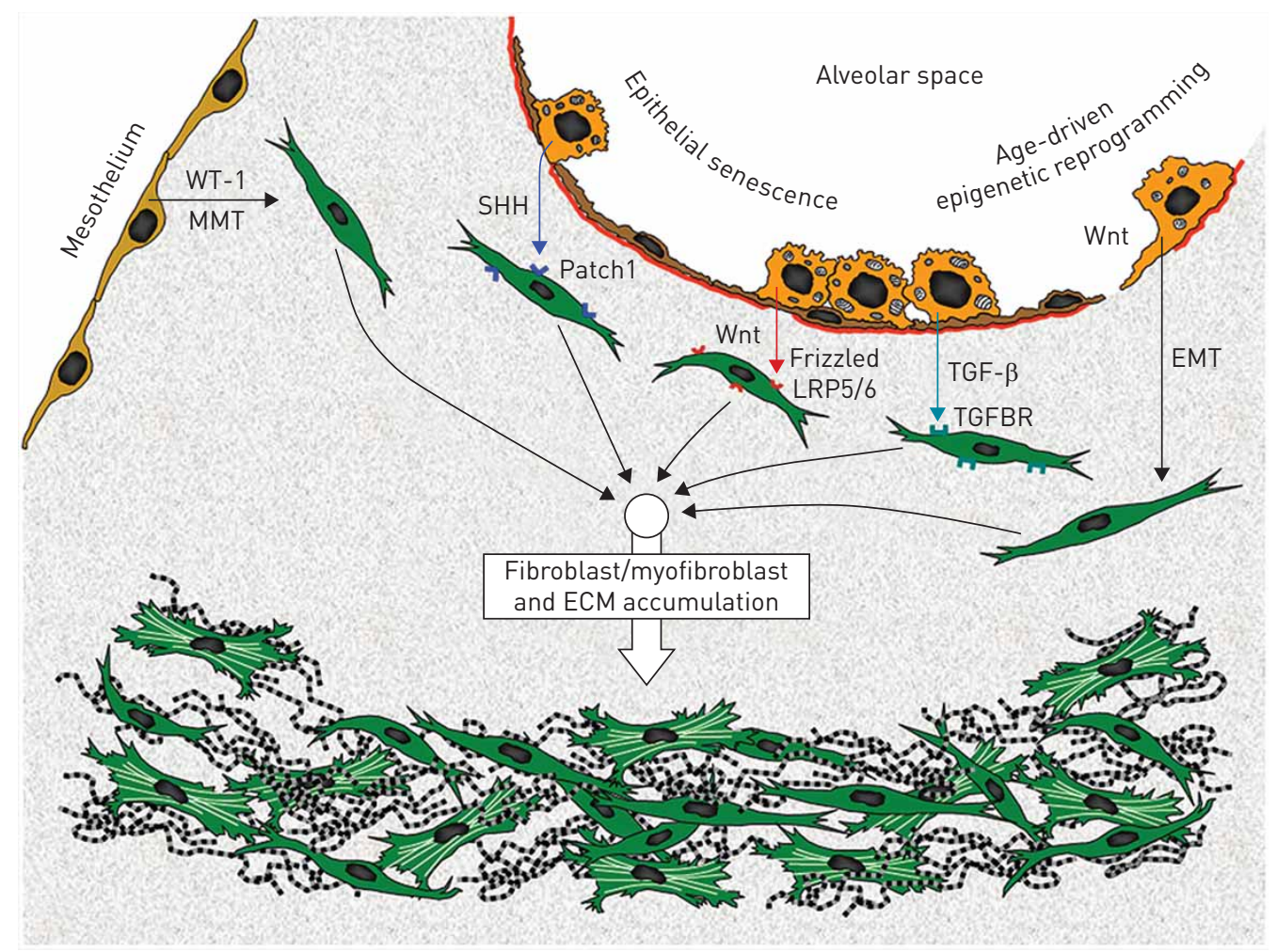

FIGURE 2 Proposed mechanisms in the pathogenesis of idiopathic pulmonary fibrosis. Aberrantly activated alveolar epithelial cells express several embryological mediators including, but not limited to, Wnt and sonic hedgehog $(\mathrm{SHH})$, which stimulate the migration and proliferation of fibroblasts and their differentiation into highly active myofibroblasts. A positive feed-back with transforming growth factor (TGF)- $\beta$ strengthens this effect. Wnt and Wilms tumour-1 (WT-1) may also contribute by autocrine stimulation to the increase of fibroblast/ myofibroblast population through epithelial-to-mesenchymal transition (EMT) or mesothelial-to-mesenchymal transition (MMT). Fibroblasts/myofibroblasts produce excessive amounts of extracellular matrix (ECM) proteins, mainly fibrillar collagens, which result in the abnormal remodelling and destruction of the lung parenchyma. TGFBR: transforming growth factor- $\beta$ receptor.

lungs is the naphthalene-induced injury in mice [30]. In this model, Club cells, specialised epithelial cells localised in distal conducting airways, are depleted 1-2 days after injury, which is followed by a rapid proliferative response leading to the complete restoration of the airway epithelium structure and function in a few days. This occurs because surviving epithelial cells reactivate developmental pathways inducing Wnt7b expression, which in turn stimulates the surrounding parabronchial smooth muscle to express FGF10, which induces epithelial repair by a transient EMT through Notch and Snail expression [32]. Moreover, a transient recapitulation of SHH and GLI1 also arises in the epithelial compartment 3 days after naphthalene injury contributing to appropriate repair [33].

Therefore, injury physiologically induces the activation of responsive signals stimulating a regenerative response executed by a transient and rigorously controlled upregulation of multiple developmental pathways including, but not limited to, Wnt/ $\beta$-catenin, SHH and FGF10 [34]. Therefore, similar developmental factors and pathways are activated in normal lung regeneration and IPF, although the final effect is diametrically opposed (table 1).

\section{Why is developmental recapitulation corrupted in IPF?}

For correct regeneration the reactivation of developmental pathways should be finely balanced with a correct temporal/spatial location to ensure normal tissue renewal. Sustained stimulation may result in aberrant epithelial behaviour and abnormal remodelling, as we propose occurs in IPF. There are at least two processes that may explain, at least partially, why the reactivation of developmental pathways may be perverted in IPF: ageing and epigenetic reprogramming. Although both processes are presented separately here, it is important to emphasise that there is a complex interplay between them. Thus, epigenetic changes contribute to ageing, while ageing is associated with an epigenetic drift characterised by gradual demethylation of the genome and hypermethylation of promoter-associated CpG islands [53]. Importantly, this age-associated epigenetic drift leads to unpredictable differences in the methylome 
Role in normal lung regeneration after injury

\section{Proposed role in IPF}

\section{Developmental pathways}

Canonical Wnt $/ \beta$-catenin

Non-canonical Wnt signalling (e.g. Wnt5a) that includes Wnt/PCP I planar cell polarityl and $\mathrm{Wnt} / \mathrm{Ca} 2$

Sonic hedgehog $(\mathrm{SHH})$

Fibroblast growth factor (FGF)9

\section{Transcription factors}

Grainyhead-like 2

Wilms tumour-1

Forkhead box F1
Regulates cell proliferation, differentiation, cell fate specification and stem cell renewal. Participates in branching morphogenesis, regional specialisation of the epithelium and mesenchyme, and the establishment of progenitor cell pools [35-38].

Wnt5a regulates epithelial-mesenchymal interactions, playing an important role in lung distal morphogenesis [39].

Indispensable for embryonic lung formation, regulates branching morphogenesis and mesenchymal proliferation affecting proliferation and differentiation of lung mesenchyme [35-38].

Regulates lung mesenchymal development. FGF9 signalling from the epithelium and reciprocal FGF10 signalling from the mesenchyme coordinate to regulate epithelial airway branching and organ size during lung embryogenesis [35-38].

Master regulator of epithelial morphogenesis and integrity including apical-basal polarity, intercellular adhesion and barrier function as well as lineage choice and differentiation of progenitor populations [21, 41].

Zinc finger transcription factor that regulates many functional properties of the developing mesothelium [42].

Is expressed at highest levels in the subepithelial lung mesenchyme between the distal bulbous part of the bud and proximal tubular part. It plays an important role in epithelium-mesenchyme signalling, as a downstream target of the $\mathrm{SHH}$ pathway. It is essential for the migration of mesenchymal cells and directly induces integrin- $\beta 3$ expression in mouse embryonic lungs, and regulates expression of VEGF signalling in endothelial cells [43]
Wnt7b is expressed by surviving ciliated epithelial cells and induces the expression of FGF10 in progenitor cells from the mesenchyme, which is essential for proliferation and differentiation during epithelial repair after lung injury [32]. ERG, an ETS transcription factor, is an essential regulator of angiogenesis and vascular stability through Wnt signalling [40].

SHH ligand and GLI1, a transcriptional target of the signalling pathway, are markedly expressed in the epithelial compartment $72 \mathrm{~h}$ after naphthalene injury [33].

Unclear. It may induce an antifibrotic phenotype in fibroblasts. In collaboration with FGF10 and other factors it may facilitate re-epithelialisation [29].
Induces epithelial cell proliferation, secretion of profibrotic mediators, epithelial-to-mesenchymal transition, fibroblast migration and myofibroblast differentiation [14-16].

Increases fibroblast proliferation and resistance to apoptosis [18].

It increases proliferation, migration, extracellular matrix production, and survival of fibroblasts. Smoothened, the obligatory signal transducer of the pathway, is required for TGF- $\beta 1$-induced myofibroblastic differentiation [20,21].

It promotes fibroblast migration and survival, but reduces fibroblast to myofibroblast differentiation [29].

May contribute to the epithelial-mesenchymal plasticity. It maintains telomerase activity in fibroblasts? [22, 23].

May increase the fibroblast/myofibroblast population inducing mesothelial-to-mesenchymal transition [25-27].

Attenuates the profibrotic phenotype in normal fibroblasts, but this effect is dampened in IPF fibroblasts [28]. 
TABLE 1 Continued

\section{Role in lung development}

It is a programmed developmental

mechanism found throughout the embryo,

including critical signalling centres in

embryonic patterning. It is regulated by the

TGF- $\beta /$ SMAD and PI3K/FOXO pathways.

Developmentally programmed senescence

is followed by macrophage infiltration,

clearance of senescent cells, and tissue

remodelling $[44,45]$.

Transforming growth factor In human embryonic stem cells, TGF- $\beta$

signalling and Smad2/3 activation are

involved in self-renewal and pluripotency.

TGF- $\beta$ is a key player for epithelial-

mesenchymal interactions during lung

morphogenesis. It regulates alveolar type 1

cell spreading and adhesion during lung

sacculation $[35,50,51]$.
Role in normal lung regeneration after injury

Unknown; it plays an important role against cancer [46].

It participates as an anti-inflammatory mediator. It has an immune suppressor effect by influencing the development, differentiation, tolerance induction and homeostasis of immune cells [52].

\section{Proposed role in IPF}

Senescence-associated secretory phenotype from the epithelial cells may contribute to the abnormal expression of the numerous profibrotic factors secreted by these cells $[2,5,47,48]$. It may contribute to the apoptosis-resistance of fibroblasts [49].

Probably the strongest profibrotic factor. It cross-talks with several developmental pathways signalling at multiple levels both during development and contributing to the aberrant integration of profibrotic factors in IPF [2]

VEGF: vascular endothelial growth factor; PI3K: phosphoinositide 3-kinase. 
among ageing individuals. In addition, some of the changes occurring during epigenetic drift may result in antagonistic pleiotropy, an evolutionary biological concept that proposes the existence of genes or processes that are beneficial early in life, but are deleterious at later stages because the force of natural selection declines with age. A subset of developmental pathways may represent a form of antagonistic pleiotropy, since they are necessary during development and for reaching adulthood, but later may cause age-related diseases [54, 55].

\section{The ageing connection}

Ageing and recapitulation of developmental pathways

Evidence suggests that ageing, and ageing-associated diseases are related to developmental signalling pathways that are perverted later in life. For example, the Wnt signalling pathway, which supports myogenic lineage progression during development, recapitulates in aged mice impairing muscle regeneration and promoting fibrosis indicating an age-related myogenic-to-fibrogenic conversion [55, 56]. Likewise, tissues from klotho-deficient mice, a genetic model of accelerated ageing, show evidence of increased Wnt signalling that triggers accelerated cellular senescence, which contributes to stem cell depletion and ageing [57]. Therefore, it can be proposed that DNA damage accumulation, shortening of telomeres and other hallmarks of ageing [3], plus an ageing-driven developmental drift are implicated in the molecular mechanisms of the ageing-associated fibrotic processes. Certainly, some of the mechanisms associated with ageing may also play a direct role in this developmental drift. For example, critical telomere shortening elicits the induction of cellular senescence, which has been recently (and surprisingly) described as a programmed developmental pathway.

\section{Cellular senescence, a hallmark of ageing, IPF and embryonic development}

Although studies on IPF lungs are scanty, recent approaches have revealed the accumulation of senescent cells, primarily in the epithelium, and less frequently in fibroblasts [47-49]. In the first study, senescent epithelial cells covering fibroblastic foci were found in IPF lungs, while they were not present in normal lungs and barely detected in COPD [47]. TGF- $\beta$ was identified as a key factor in the p53-independent induction of $\mathrm{p} 21$, a mechanism that is reminiscent of developmental senescence. Importantly, senescent cells secreted increased amounts of interleukin (IL)-1 $\beta$, which was sufficient to induce fibroblast-to-myofibroblast differentiation [47]. The presence of epithelial senescence in areas of active fibrosis was corroborated 2 years later [48]. These cells also exhibited markers of insufficient autophagy, and in vitro models revealed that autophagy inhibition was sufficient to induce an increased rate of epithelial cell senescence. More recently, the expression of p16 and p21 was also detected, in addition to in alveolar epithelial cells, in fibroblasts/myofibroblasts within the fibroblastic foci indicating that these cells also display a senescent phenotype [49]. Senescent fibroblasts were mostly non-proliferative and showed little evidence of apoptosis, which may contribute to their impaired elimination. Taken together, these findings suggest that senescence may contribute to the pathogenesis of IPF through dual mechanisms, causing the abnormal secretory pattern of the lung epithelium and increasing the resistance to apoptosis in myofibroblasts. In addition, some "systems senescence" has been described in this disease, such as immunosenescence and endocrine-senescence [46, 58]. Immunosenescence is characterised by an age-related functional decline affecting the innate and adaptive arms of immune function and promoting an increased susceptibility to infection, cancer and autoimmune disorders [59]. Senescent subsets of T-lymphocytes such as $\mathrm{CD}^{+} \mathrm{CD} 28$ (null) T-cells are increased in IPF lungs and peripheral blood [58]. Likewise, an exaggerated decline of dehydroepiandrosterone, a steroid prohormone that decreases physiologically with age, has been revealed in IPF patients [46]. However, the role of "systems senescence" in the development of IPF is uncertain, but may contribute to different rates of progression; in fact, some features of immunosenescence seem to be associated with worse clinical outcome [58].

Senescent cells are characterised by a stable cell-cycle arrest, resistance to apoptosis and a multifaceted senescence-associated secretory phenotype (SASP) that yields widespread signalling to the external environment [59]. Triggers of ageing-associated senescence include DNA damage, loss of telomere protective functions and oncogenes that signal through various pathways, converging in the activation of the cyclin-dependent kinase inhibitors p16, p15, p21 and p27 $[6,59,60]$. Senescence provokes age-related dysfunction decreasing the regenerative potential by creating a persistent growth arrest, and secreting multiple mediators that disturb tissue structure and organisation.

Surprisingly, two recent studies identified senescent cells in multiple locations during mammalian embryonic development and demonstrated that during normal development, programmed cell senescence contributes to tissue remodelling and morphogenesis, probably eliminating transient structures and regulating the relative abundance of different cell populations [44, 45]. Developmental senescence appears during particular time windows, occurs in major signalling centres that instruct growth and patterning, and disappears before birth. Gene expression profiles of the senescent cells showed that the signalling 
pathways that regulate developmentally programmed senescence included TGF- $\beta$, Hedgehog, and Wnt [44]. In addition, programmed developmental senescence shares a molecular signature with oncogene-induced senescence including the expression of p21, p15 and a number of SASP components [45]. Remarkably, many of the secreted proteins that are common in developmental and stress-induced senescence involve developmental pathways, suggesting that some of the paradoxical functions of the SASP in adult senescence, including age-associated diseases such as IPF, might implicate the reactivation of developmental processes. The common expression of crucial factors suggests that senescence might primarily have arisen as a programmed developmental mechanism that was later adapted during evolution to carry out its adult roles [45]. In this context, the process of cellular senescence may also be considered as an example of antagonistic pleiotropy or deleterious developmental drift, since initially it has beneficial roles in development (and possibly in physiological repair), but then is altered qualitatively or quantitatively contributing to pathological repair in IPF. However, the difference with classical examples of antagonistic pleiotropy is that senescence that recapitulates later in adult life has also a beneficial effect, since it represents a crucial mechanism in tumour suppression [59].

In this context, it is important to emphasise that ageing-associated senescence may have both beneficial and detrimental effects in tissue remodelling [60,61]. Thus, while senescence seems to attenuate the fibrotic response in many organs, e.g. liver, kidneys, heart and skin, it seems to be deleterious in IPF. The difference may be related with the main affected cell type. Thus, while in the tissues where the process seems to be beneficial, senescence occurs in myofibroblasts [60], in IPF senescence occurs primarily in epithelial cells (figure 3) [47, 48].

\section{The epigenetic connection}

An important regulation of gene and phenotypic expression occurs at the level of the epigenome, the molecular interface between the genome and the environment. The epigenetic machinery covers multiple levels of regulation, including DNA methylation, post-translational histone modifications and non-coding RNAs. The epigenome is influenced by environmental factors and collectively shapes the transcriptional activity of genes without changing the underlying genetic code [62]. Recent evidence indicates that changes in DNA methylation and deregulation of microRNAs (miRNAs) may contribute to the recapitulation of developmental pathways in IPF lungs.

\section{DNA methylation reprogramming and reactivation of developmental pathways in IPF}

The machinery responsible for maintaining normal DNA methylation patterns becomes gradually and stochastically deregulated with age (epigenetic drift) $[63,64]$. DNA methylation changes are bidirectional with both hyper- and hypo-methylations occurring at comparable rates, with random errors in methylation maintenance, probably because the fidelity of transmission of epigenetic patterns is variable across the genome [64].

A recent study involving 94 patients with IPF and 67 controls identified 2130 genome-wide differentially methylated regions, of which 738 were associated with significant changes in gene expression and enriched for an expected inverse relationship between methylation and gene expression [65]. Among the most enriched canonical pathways was the $\mathrm{Wnt} / \mathrm{\beta}$-catenin signalling pathway. Likewise, the evaluation of binding motifs in promoters of the 738 differentially expressed genes revealed the increase of several

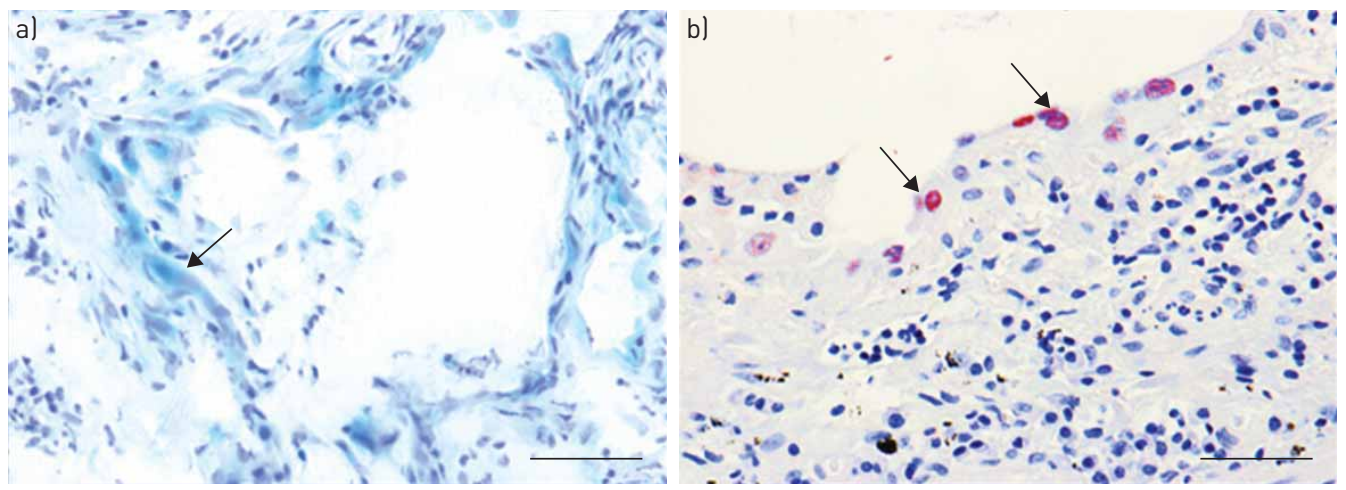

FIGURE 3 Premature senescence of alveolar epithelial cells in idiopathic pulmonary fibrosis lungs. a) Senescence-associated- $\beta$-galactosidase activity is observed in several alveolar epithelial cells (arrow). b) Expression of $\mathrm{p} 21$. The signal is located to the nuclei of several epithelial cells (arrows). Scale bars $=50 \mu \mathrm{m}$. Image courtesy of David Medina (Facultad de Ciencias, UNAM, Mexico city, Mexico). 
regulators of lung development, such as $\beta$-catenin and FOXC2, a gene that participates in mesenchymal tissue development. Protein-protein network analysis also identified a number of genes related to development such as FZD8, GLI3, JAG2, Wnt5B and Wnt10A.

Curiously, castor zinc finger 1 (CASZ1), a para-zinc-finger transcription factor showed multiple differentially methylated regions. CASZ1 is required for vertebrate heart development [66], blood vessel assembly and lumen morphogenesis [67], and is dynamically expressed during neurogenesis [68]. Interestingly, while there was a marked decrease of CASZ1 expression in airway epithelial cells, alveolar epithelial cells in fibrotic areas expressed higher concentrations of this protein.

Therefore, this study revealed that numerous changes in DNA methylation contribute to the recapitulation of developmental pathways in IPF lungs.

\section{Non-coding microRNAs}

Non-coding miRNAs have emerged as relevant transcriptional regulators in both physiological and pathological conditions. Although miRNAs constitute only $3 \%$ of the human genome, it is estimated that they regulate $\sim 90 \%$ of genes, silencing them through degradation of target mRNA or inhibition of protein translation [69]. Recent studies have linked dysregulated miRNA function to a range of processes associated with ageing and ageing-related diseases and additionally, crucial senescence-associated molecules such as 21 and p16 are modulated by miRNAs [61, 70-72].

Studies in IPF lungs have identified significant changes in $\sim 10 \%$ of miRNAs with a potential pathogenic role in the disease (figure 4) $[73,74]$. Remarkably, many of the dysregulated miRNAs in IPF lungs were similarly expressed in embryonic lungs [75]. Importantly, several deregulated miRNAs target developmental pathways [73, 74]. For example, the increase of miR-154 and the decrease of miR-30a, miR-30d and miR-92a observed in IPF lungs may result in the upregulation of the Wnt signalling pathway $[75,76]$. The miR-154 family inhibits several Wnt pathway repressors, e.g. dickkopf and Wnt signalling pathway inhibitor 2 , and increases several Frizzled receptors, $\beta$-catenin, and WISP1, leading to a largely activation of the Wnt/ $\beta$-catenin pathway [75]. Moreover, the effects of miR-154 on fibroblasts were reversed by inhibition of the $\mathrm{Wnt} / \beta$-catenin pathway. Likewise, miR-92a was found to be remarkably decreased in IPF lungs, which was accompanied by increased WISP1 protein expression. A miR-92a binding site was identified on the WISP1 $3^{\prime}$-untranslated region (UTR) in an argonaute binding site indicating that the decrease of this miRNA contributes to the increased Wnt pathway [76].

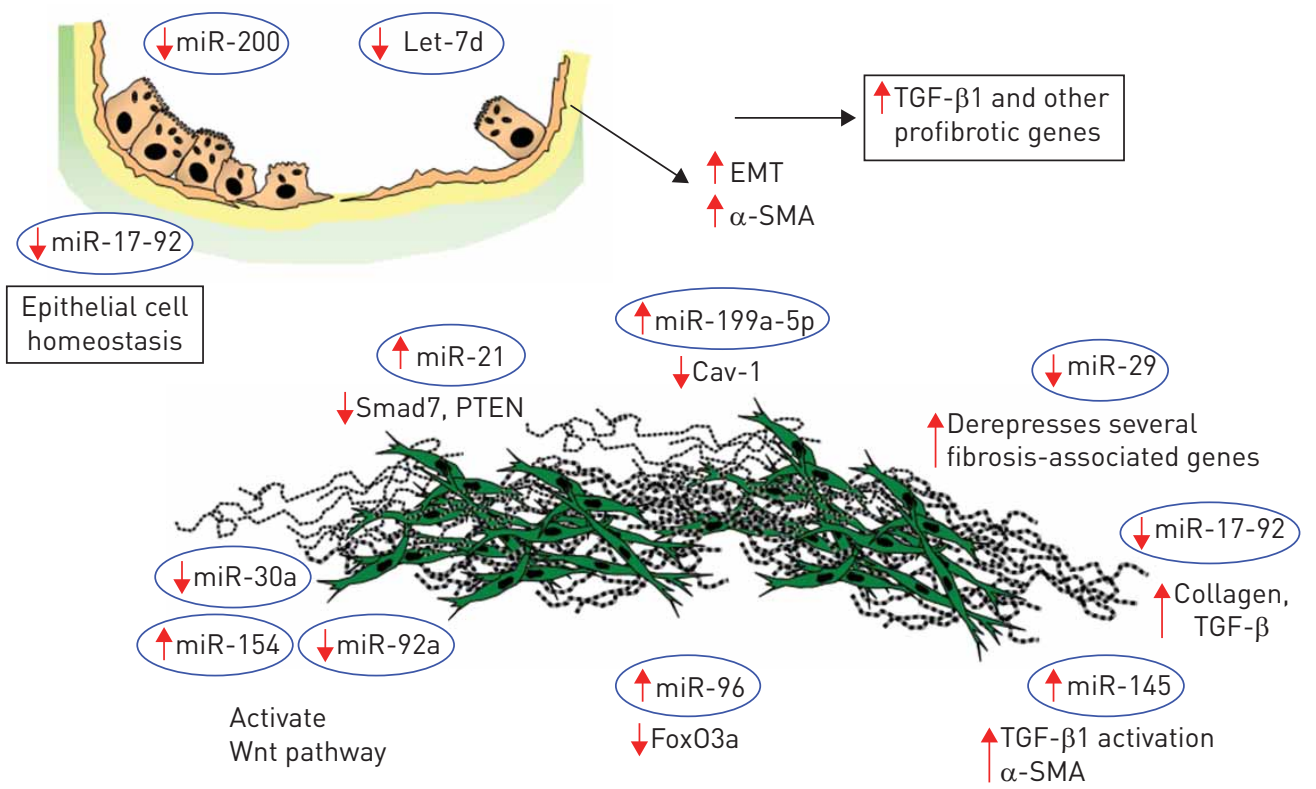

FIGURE 4 Deregulated microRNAs play a role in the development and progression of idiopathic pulmonary fibrosis. The schematics illustrate some of the up- or down-regulated microRNAs that affect epithelial cells and fibroblasts switching them to profibrotic phenotypes. TGF: transforming growth factor; EMT: epithelial-to-mesenchymal transition; $\alpha$-SMA: $\alpha$-smooth muscle actin; PTEN: phosphatase and tensin homologue; Cav-1: caveolin-1. 


\section{Therapeutic approaches for IPF}

Despite substantial progress in our knowledge about the pathogenesis of IPF, the mechanisms underlying its biopathology remain unclear and the therapeutic options are very limited. Until recently, with the notion that IPF was an inflammation driven disorder, corticosteroids either alone or combined with different immunosuppressive drugs were the mainstay for its therapy. However, a recent clinical trial evaluating a potent anti-inflammatory therapy revealed that this approach was not only ineffective but also harmful increasing the incidence of hospitalisations and death [77].

Recently, two antifibrotic drugs, pirfenidone and nintedanib, were shown to decrease the rate of decline in forced vital capacity in IPF patients with mild-to-moderate functional impairment [78, 79]. The mechanisms by which pirfenidone may reduce the fibrotic response in human disease are uncertain. Some studies suggest that pirfenidone downregulates some profibrotic growth factors, including TGF- $\beta$, and scavenging of reactive oxygen species [80]. Nintedanib is a multiple tyrosine kinase inhibitor directed against platelet-derived growth factor, vascular endothelial growth factor and FGF [80]. To our knowledge, these drugs have no effect on developmental pathways although some tyrosine kinase inhibitors may reduce senescence (discussed later in this review). Additionally, nintedanib may affect some developmental pathways through the inhibition of FGF receptors 1, 2 and 3, which are required throughout embryonic development. Importantly however, patients with moderate-to-severe functional alterations were excluded from these trials, and consequently, many patients seen in clinical practice have not been evaluated. Therefore, a curative therapy for this devastating lung disease is still required.

\section{Developmental pathways as therapeutic targets}

Emerging evidence indicates that targeting $\mathrm{Wnt} / \beta$-catenin attenuates the lung fibrotic response in experimental models. For example, ICG-001, a small molecule that selectively interacts with CREB-binding protein blocking the $\beta$-catenin/CBP interface, prevents and even reverses established fibrosis in bleomycin-induced lung injury reducing the expression of many profibrotic genes [81]. Similar results were reported with XAV939, a small-molecule compound that stabilises the axin2 structure leading to the degradation of $\beta$-catenin and the suppression of Wnt/ $\beta$-catenin signalling [82]. XAV939 reduced the severity of lung fibrosis induced by bleomycin and increased mouse survival. Likewise, NSC668036, which binds to the dishevelled PDZ domain inhibiting Wnt signalling and blocking PDZ-mediated interactions, attenuated the severity of bleomycin-induced pulmonary fibrosis [83]. Inhibition of the activity of WISP1 using repetitive orotracheal applications of specific neutralising antibodies was also effective to attenuate bleomycin-induced lung fibrosis [15].

Recent work also suggests that inhibition of GLI transcription factors could also modulate the fibrotic process. GANT61 (Gli-ANTagonist), a low molecular weight compound, blocks the hedgehog pathway inhibiting the binding of GLI1 and GLI2 to their DNA targets [84]. In bleomycin-induced pulmonary fibrosis GANT61 strongly reduced the extent of lung lesions and decreased lung collagen content [85]. A variety of Wnt and SHH signalling inhibitors are currently in development and some of them are even under investigation in clinical trials, primarily in cancer, giving us promising therapeutic options for IPF in the future. For example, there are several clinical trials exploring the safety and efficacy of Vismodegib (GDC-0449), an inhibitor of the hedgehog pathway, in some types of cancer [86-88].

One of the challenges to address for an effective pathway-based IPF therapy is how to disrupt the many altered fibrogenic pathways. Since one specific miRNA may target multiple genes potentially implicated in fibrogenesis, miRNAs are attractive targets for molecular therapy.

In this context, a growing body of evidence suggests that modulation of miRNAs either blocking upregulated fibrogenic miRNAs (fibromiRs) or restoring downregulated antifibrogenic miRNAs (antifibromiRs) may represent a potential therapeutic approach for IPF. For instance, administration of antisense probes of miR-21 (an upregulated fibromiR in IPF) attenuated the severity of experimental lung fibrosis, even when treatment was given several days after injury [89]. In another study, it was demonstrated that several members of the miR-200 family (mainly localised to the alveolar epithelium) are downregulated in the lungs of mice with bleomycin-induced lung fibrosis and of patients with IPF. Interestingly, bleomycin-instilled mice that received miR-200c mimics exhibited remarkably attenuated extent of lung fibrotic lesions [90]. Likewise, gene transfer of the antifibrotic miR-29b through the Sleeping Beauty SB-transposon system as well as therapeutic treatment with miR-29b mimics reversed bleomycin-established lung fibrosis in mice [91, 92]. Similarly, miR-326, which targets the TGF- $\beta 13^{\prime}$ UTR, is downregulated in IPF and during progression of lung fibrosis, and intranasal delivery of miR-326 mimics reduced lung TGF- $\beta 1$ levels and decreased the functional and morphological features of bleomycin-induced fibrosis [93].

Some anti-miRNAs are currently being tested in clinical trials in non-lung diseases. For example, miravirsen, which targets miR-122, has been used in patients with chronic hepatitis $\mathrm{C}$ resulting in a prolonged decrease of hepatitis C virus RNA levels [94]. 
All these findings indicate that targeting specific pathogenic miRNAs is feasible in vivo and might be a future therapeutic target in IPF. The main challenges in miRNA-based therapy include the selectivity of the miRNA, the redundancy of the miRNAs, and the specificity of the delivery approach. To solve this problem, a large-scale mammalian gene circuit serving as an assay for drug discovery against miRNA targets was recently described. This logic circuit may allow the accurate identification of specific target modulators with high throughput [95].

\section{Can cellular senescence be reversed?}

Hypothetically, and assuming that premature and persistent alveolar epithelial cell and fibroblast senescence is an important pathogenic mechanism in IPF, "anti-senescence" therapies may represent an attractive option. In this context, several potential pharmacological agents that may inhibit the major senescence-regulatory pathways have been evaluated. For example, it was demonstrated that treatment with rupatadine protected against the progression of fibrosis and improved lung function in mice and rats injured with bleomycin or silica [96]. This effect was mechanistically associated with the interruption of p53/21-dependent premature epithelial senescence.

Likewise, mTOR inhibition with rapamycin decreases epithelial stem cell depletion preventing cells from entering senescence and terminal differentiation programmes [97]. Rapamycin provoked a remarkable decrease in the formation of reactive oxygen species suggesting that inhibition of oxidative stress may represent one of the mechanisms by which it protects from cell senescence and stem cell depletion. Rapamycin is currently used in humans with several pathological conditions, e.g. for the treatment of lymphangioleiomyomatosis, a rare multisystem disease affecting predominantly young women [98], and a randomised double-blind clinical trial with rapamycin in IPF is currently ongoing [99].

In the same line of thought, pharmacological or genetic targeting of superoxide-producing NADPH oxidases have also been proven. Thus, GKT137831, a small-molecule Nox1/Nox4 dual inhibitor, attenuated the senescent myofibroblast phenotype leading to a reversal of persistent lung fibrosis in aged mice [49]. This drug has also been shown to be protective in mice with pre-existing diabetes and established kidney disease, in rats with ischaemic retinopathy, and in cardiac fibrosis [100-102].

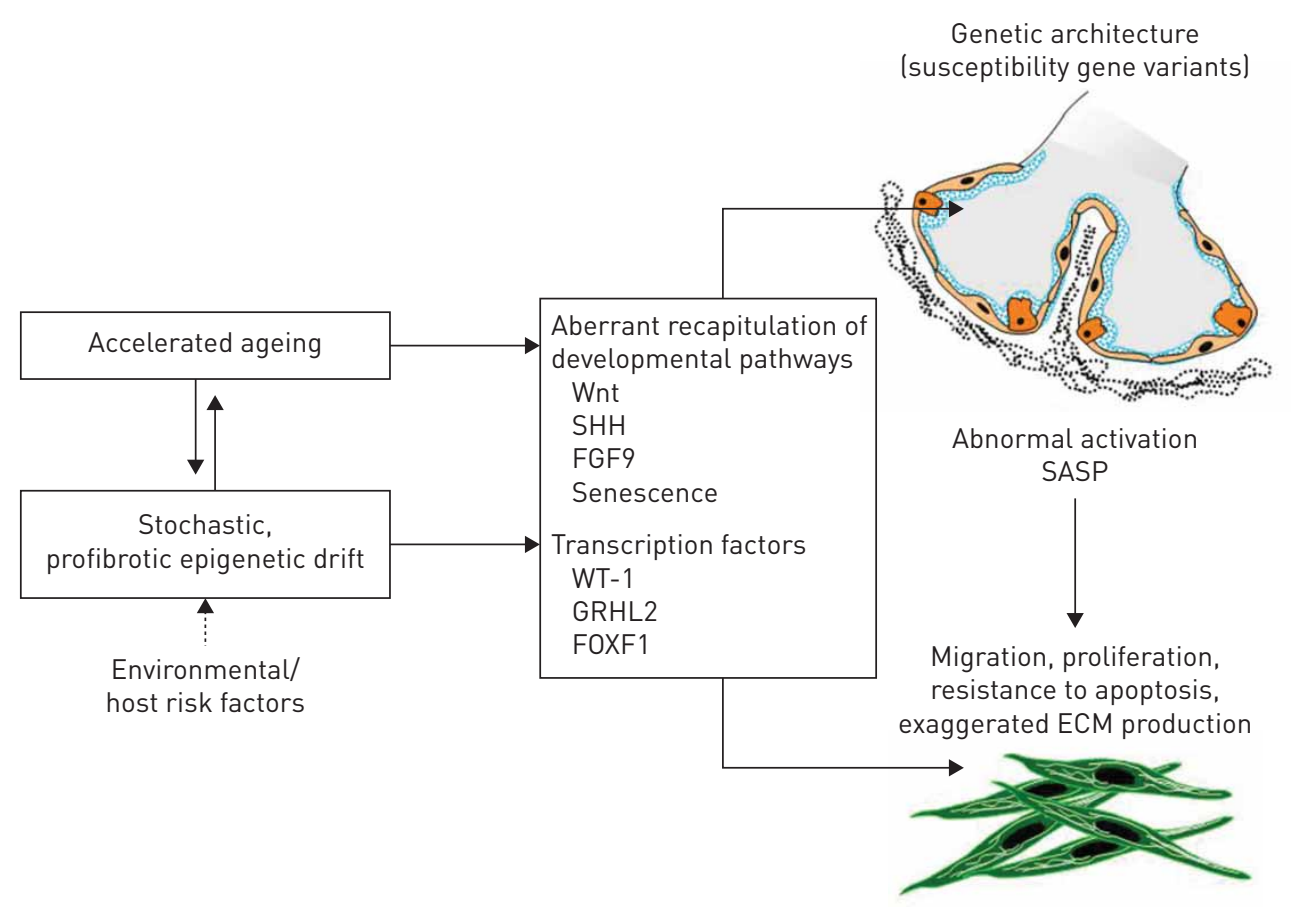

FIGURE 5 Proposed integral model for the development of idiopathic pulmonary fibrosis. A complex interplay between ageing-associated mechanisms le.g. abnormal shortening of telomeres and mitochondrial dysfunction) and a stochastic profibrotic epigenetic developmental drift results in the aberrant recapitulation and persistence of embryological pathways. Some environmental factors (e.g. smoking) and host factors (e.g. nutritional state) also contribute to accelerated ageing and to the epigenetic drift. These processes, together with a certain genetic susceptibility involving a set of gene variants that affect primarily the epithelial integrity, result in the abnormal activation of epithelial cells and fibroblasts. SHH: sonic hedgehog; FGF: fibroblast growth factor; WT1: Wilms tumour-1; GRHL2: grainyhead-like 2; FOXF1: forkhead box F1; SASP: senescence-associated secretory phenotype; ECM: extracellular matrix. 
Studies with antioxidant drugs in IPF are scarce, but a recently reported clinical trial showed that $\mathrm{N}$-acetylcysteine, a drug known to decrease oxidative stress, had no benefit in patients with moderate functional impairment [103].

Dasatinib and quercetin have showed particular promise in clearing senescent cells [104]. Dasatinib is an inhibitor of multiple tyrosine kinases, used for treating cancers. In vivo, this combination reduced the senescent cell burden in chronologically aged and progeroid mice, improved cardiac function and reduced dysfunction caused by localised irradiation [104]. Moreover, intermittent drug administration extended the health span of progeroid mice, delaying age-related symptoms and pathology. In the same line of thought, ABT263 (a potent inducer of apoptosis) was identified as a selective and potent killer of fibroblasts and epithelial senescent cells. Moreover, oral administration of ABT263 to normally aged mice or mice with prematurely induced ageing depleted senescent cells and this clearance rejuvenated the prematurely aged haematopoietic system and senescent muscle stem cells [105].

Recent experimental data have demonstrated that fibroblasts from accelerated and chronological ageing donors, characterised by premature senescence entry, exhibit a strong upregulation of nuclear factor $(\mathrm{NF})-\kappa \mathrm{B}$ signalling. This activation impairs the generation of induced pluripotent stem cells, which demonstrates that NF- $\mathrm{KB}$ constitutes a novel age-associated cell reprogramming barrier [106, 107]. Mechanistically, NF- $\mathrm{BB}$ signalling elicits the expression of some reprogramming repressors, such as the histone methyltransferase DOT1L, and enhances premature senescence in aged cells. Accordingly, it was shown that NF- $\mathrm{KB}$ or DOT1L inhibition increased reprogramming efficiency of aged fibroblasts, without affecting fibroblasts from young donors. Importantly, treatment of Zmpste24-deficient mice, a well-studied animal model of accelerated ageing, during their lifetime with DOT1L inhibitors increased longevity and reversed progeroid phenotypes.

These results illustrate the possibilities and difficulties in reprogramming cells from pathological processes characterised by premature senescence, supporting the interest in development of intervention strategies to enhance cell reprogramming and plasticity.

\section{Conclusions}

IPF is a devastating lung disorder with poor prognosis and marginal therapeutic options. Signalling cascades and cellular processes that coordinate the critical stages of lung development are reactivated post-developmentally, under rigorous control, for proper regenerative responses after injury. In IPF, however, some of these embryological pathways and processes recapitulate without regulation resulting in an aberrant repair programme (figure 5). Moreover, these developmental cascades interact with one another and crosstalk with TGF- $\beta$, a central profibrotic mediator, probably provokes a complex positive-feedback loop. The aberrant recapitulation of many of these developmental pathways is driven by ageing, through a developmental epigenetic drift, that impairs the generation of normal wound healing. Specifically, this theoretical approach indicates that IPF is steered, at least partially, by developmental pathways that get twisted later in life.

A better understanding of the role of diverse embryological signalling pathways involved in IPF may open novel therapeutic opportunities for this lethal disease. In this context, some promising results have been found in preclinical models underscoring the likelihood of developing therapeutic strategies to target these pathways. However, translation of these findings into clinical trials faces important challenges primarily related to the complexity of selectively delivering the drugs to the target cells to avoid potential deleterious effects from manipulating such essential pathways.

\section{References}

1 Raghu G, Rochwerg B, Zhang Y, et al. An official ATS/ERS/JRS/ALAT clinical practice guideline: treatment of idiopathic pulmonary fibrosis. An update of the 2011 clinical practice guideline. Am J Respir Crit Care Med 2015; 192: e3-e19.

2 King TE Jr, Pardo A, Selman M. Idiopathic pulmonary fibrosis. Lancet 2011; 378: 1949-1961. López-Otín C, Blasco MA, Partridge L, et al. The hallmarks of aging. Cell 2013; 153: 1194-1217.

Meiners S, Eickelberg O, Königshoff M. Hallmarks of the ageing lung. Eur Respir J 2015; 45: 807-827.

Selman M, Pardo A. Revealing the pathogenic and aging-related mechanisms of the enigmatic idiopathic pulmonary fibrosis. An integral model. Am J Respir Crit Care Med 2014; 189: 1161-1172.

6 Alder JK, Chen JJ, Lancaster L, et al. Short telomeres are a risk factor for idiopathic pulmonary fibrosis. Proc Natl Acad Sci USA 2008; 105: 13051-13056.

7 Demopoulos K, Arvanitis DA, Vassilakis DA, et al. MYCL1, FHIT, SPARC, p16(INK4) and TP53 genes associated to lung cancer in idiopathic pulmonary fibrosis. J Cell Mol Med 2002; 6: 215-222.

8 Bueno M, Lai YC, Romero Y, et al. PINK1 deficiency impairs mitochondrial homeostasis and promotes lung fibrosis. J Clin Invest 2015; 125: 521-538.

9 Korfei M, von der Beck D, Henneke I, et al. Comparative proteome analysis of lung tissue from patients with idiopathic pulmonary fibrosis (IPF), non-specific interstitial pneumonia (NSIP) and organ donors. J Proteomics 2013; 85: 109-128. 
Mercado N, Ito K, Barnes PJ. Accelerated ageing of the lung in COPD: new concepts. Thorax 2015; 70: 482-489. Selman M, Pardo A, Kaminski N. Idiopathic pulmonary fibrosis: aberrant recapitulation of developmental programs? PLoS Med 2008; 5: e62.

Selman M, Pardo A, Barrera L, et al. Gene expression profiles distinguish idiopathic pulmonary fibrosis from hypersensitivity pneumonitis. Am J Respir Crit Care Med 2006; 173: 188-198.

Chilosi M, Poletti V, Zamò A, et al. Aberrant Wnt/beta-catenin pathway activation in idiopathic pulmonary fibrosis. Am J Pathol 2003; 162: 1495-1502.

Königshoff M, Balsara N, Pfaff EM, et al. Functional Wnt signaling is increased in idiopathic pulmonary fibrosis. PLoS One 2008; 3: e2142.

Königshoff M, Kramer M, Balsara N, et al. WNT1-inducible signaling protein-1 mediates pulmonary fibrosis in mice and is upregulated in humans with idiopathic pulmonary fibrosis. J Clin Invest 2009; 119: 772-787.

Meuten T, Hickey A, Franklin K, et al. WNT7B in fibroblastic foci of idiopathic pulmonary fibrosis. Respir Res 2012; 13: 62 .

Oda K, Yatera K, Izumi H, et al. Profibrotic role of WNT10A via TGF- $\beta$ signaling in idiopathic pulmonary fibrosis. Respir Res 2016; 17: 39.

Vuga LJ, Ben-Yehudah A, Kovkarova-Naumovski E, et al. WNT5A is a regulator of fibroblast proliferation and resistance to apoptosis. Am J Respir Cell Mol Biol 2009; 41: 583-589.

Zhou B, Liu Y, Kahn M, et al. Interactions between $\beta$-catenin and transforming growth factor- $\beta$ signaling pathways mediate epithelial-mesenchymal transition and are dependent on the transcriptional co-activator cAMP-response element-binding protein (CREB)-binding protein (CBP). J Biol Chem 2012; 287: 7026-7038.

Cigna N, Farrokhi Moshai E, Brayer S, et al. The hedgehog system machinery controls transforming growth factor- $\beta$-dependent myofibroblastic differentiation in humans: involvement in idiopathic pulmonary fibrosis. Am J Pathol 2012; 181: 2126-2137.

Bolaños AL, Milla CM, Lira JC, et al. Role of Sonic Hedgehog in idiopathic pulmonary fibrosis. Am J Physiol Lung Cell Mol Physiol 2012; 303: L978-L990.

Varma S, Mahavadi P, Sasikumar S, et al. Grainyhead-like 2 (GRHL2) distribution reveals novel pathophysiological differences between human idiopathic pulmonary fibrosis and mouse models of pulmonary fibrosis. Am J Physiol Lung Cell Mol Physiol 2014; 306: L405-L419.

Chen W, Dong Q, Shin KH, et al. Grainyhead-like 2 enhances the human telomerase reverse transcriptase gene expression by inhibiting DNA methylation at the $5^{\prime}$-CpG island in normal human keratinocytes. $J$ Biol Chem 2010; 285: 40852-40863.

Liu T, Ullenbruch M, Young Choi Y, et al. Telomerase and telomere length in pulmonary fibrosis. Am J Respir Cell Mol Biol 2013; 49: 260-268.

Batra H, Antony VB. The pleural mesothelium in development and disease. Front Physiol 2014; 5: 284.

Zolak JS, Jagirdar R, Surolia R, et al. Pleural mesothelial cell differentiation and invasion in fibrogenic lung injury. Am J Pathol 2013; 182: 1239-1247.

Sontake V, Shanmukhappa SK, DiPasquale BA, et al. Fibrocytes regulate Wilms tumor 1-positive cell accumulation in severe fibrotic lung disease. J Immunol 2015; 195: 3978-3991.

Melboucy-Belkhir S, Pradère P, Tadbiri S, et al. Forkhead Box F1 represses cell growth and inhibits COL1 and ARPC2 expression in lung fibroblasts in vitro. Am J Physiol Lung Cell Mol Physiol 2014; 307: L838-L847.

Joannes A, Brayer S, Besnard V, et al. FGF9 and FGF18 in idiopathic pulmonary fibrosis promote survival and migration and inhibit myofibroblast differentiation of human lung fibroblasts in vitro. Am J Physiol Lung Cell Mol Physiol 2016; 310: L615-L629.

Inoue Y, King TE Jr, Barker E, et al. Basic fibroblast growth factor and its receptors in idiopathic pulmonary fibrosis and lymphangioleiomyomatosis. Am J Respir Crit Care Med 2002; 166: 765-773.

Marchand-Adam S, Plantier L, Bernuau D, et al. Keratinocyte growth factor expression by fibroblasts in pulmonary fibrosis: poor response to interleukin-1ß. Am J Respir Cell Mol Biol 2005; 32: 470-477.

Volckaert T, Dill E, Campbell A, et al. Parabronchial smooth muscle constitutes an airway epithelial stem cell niche in the mouse lung after injury. J Clin Invest 2011; 121: 4409-4419.

Watkins DN, Berman DM, Burkholder SG, et al. Hedgehog signalling within airway epithelial progenitors and in small-cell lung cancer. Nature 2003; 422: 313-317.

Sun G, Irvine KD. Control of growth during regeneration. Curr Top Dev Biol 2014; 108: 95-120.

Volckaert T, De Langhe SP. Wnt and FGF mediated epithelial-mesenchymal crosstalk during lung development. Dev Dyn 2015; 244: 342-366.

Warburton D, El-Hashash A, Carraro G, et al. Lung organogenesis. Curr Top Dev Biol 2010; 90: 73-158. Swarr DT, Morrisey EE. Lung endoderm morphogenesis: gasping for form and function. Annu Rev Cell Dev Biol 2015; 31: 553-573.

McCulley D, Wienhold M, Sun X. The pulmonary mesenchyme directs lung development. Curr Opin Genet Dev 2015; 32: 98-105.

Li C, Xiao J, Hormi K, et al. Wnt5a participates in distal lung morphogenesis. Dev Biol 2002; $248:$ 68-81.

Birdsey GM, Shah AV, Dufton N, et al. The endothelial transcription factor ERG promotes vascular stability and growth through Wnt/ $\beta$-catenin signaling. Dev Cell 2015; 32: 82-96.

Varma S, Cao Y, Tagne JB, et al. The transcription factors Grainyhead-like 2 and NK2-homeobox 1 form a regulatory loop that coordinates lung epithelial cell morphogenesis and differentiation. J Biol Chem 2012; 287: 37282-37295.

Cano E, Carmona R, Muñoz-Chápuli R. Wt1-expressing progenitors contribute to multiple tissues in the developing lung. Am J Physiol Lung Cell Mol Physiol 2013; 305: L322-L332.

Dharmadhikari AV, Szafranski P, Kalinichenko VV, et al. Genomic and epigenetic complexity of the FOXF1 locus in 16q24.1: implications for development and disease. Curr Genomics 2015; 16: 107-116.

Muñoz-Espín D, Cañamero M, Maraver A, et al. Programmed cell senescence during mammalian embryonic development. Cell 2013; 155: 1104-1118.

Storer M, Mas A, Robert-Moreno A, et al. Senescence is a developmental mechanism that contributes to embryonic growth and patterning. Cell 2013; 155: 1119-1130.

Mendoza-Milla C, Valero Jiménez A, Rangel C, et al. Dehydroepiandrosterone has strong antifibrotic effects and is decreased in idiopathic pulmonary fibrosis. Eur Respir J 2013; 42: 1309-1321. 
Minagawa S, Araya J, Numata T, et al. Accelerated epithelial cell senescence in IPF and the inhibitory role of SIRT6 in TGF- $\beta$-induced senescence of human bronchial epithelial cells. Am J Physiol Lung Cell Mol Physiol 2011; 300: L391-L401.

Araya J, Kojima J, Takasaka N, et al. Insufficient autophagy in idiopathic pulmonary fibrosis. Am J Physiol Lung Cell Mol Physiol 2013; 304: L56-L69.

Hecker L, Logsdon NJ, Kurundkar D, et al. Reversal of persistent fibrosis in aging by targeting Nox4-Nrf2 redox imbalance. Sci Transl Med 2014; 6: 231ra47.

Wang Y, Frank DB, Morley MP, et al. HDAC3-dependent epigenetic pathway controls lung alveolar epithelial cell remodeling and spreading via miR-17-92 and TGF- $\beta$ signaling regulation. Dev Cell 2016; 36: 303-315.

Itoh F, Watabe T, Miyazono K. Roles of TGF- $\beta$ family signals in the fate determination of pluripotent stem cells. Semin Cell Dev Biol 2014; 32: 98-106.

Sheng J, Chen W, Zhu HJ. The immune suppressive function of transforming growth factor- $\beta$ (TGF- $\beta$ ) in human diseases. Growth Factors 2015; 33: 92-101.

Zampieri M, Ciccarone F, Calabrese R, et al. Reconfiguration of DNA methylation in aging. Mech Ageing Dev 2015; 151: 60-70.

de Magalhães JP, Sandberg A. Cognitive aging as an extension of brain development: a model linking learning, brain plasticity, and neurodegeneration. Mech Ageing Dev 2005; 126: 1026-1033.

Lezzerini M, Smith RL, Budovskaya Y. Developmental drift as a mechanism for aging: lessons from nematodes. Biogerontology 2013; 14: 693-701.

Brack AS, Conboy MJ, Roy S, et al. Increased Wnt signaling during aging alters muscle stem cell fate and increases fibrosis. Science 2007; 317: 807-810.

Liu H, Fergusson MM, Castilho RM, et al. Augmented Wnt signaling in a mammalian model of accelerated aging. Science 2007; 317: 803-806.

Gilani SR, Vuga LJ, Lindell KO, et al. CD28 down-regulation on circulating CD4 T-cells is associated with poor prognoses of patients with idiopathic pulmonary fibrosis. PLoS One 2010; 5: e8959.

Campisi J. Aging, cellular senescence, and cancer. Annu Rev Physiol 2013; 75: 685-705.

Muñoz-Espín D, Serrano M. Cellular senescence: from physiology to pathology. Nat Rev Mol Cell Biol 2014; 15 482-496.

Abdelmohsen K, Gorospe M. Noncoding RNA control of cellular senescence. Wiley Interdiscip Rev RNA 2015; 6 : 615-629.

Kanherkar RR, Bhatia-Dey N, Csoka AB. Epigenetics across the human lifespan. Front Cell Dev Biol 2014; 2: 49.

Teschendorff AE, West J, Beck S. Age-associated epigenetic drift: implications, and a case of epigenetic thrift? Hum Mol Genet 2013; 22: R7-R15.

Issa JP. Aging and epigenetic drift: a vicious cycle. J Clin Invest 2014; 124: 24-29.

Yang IV, Pedersen BS, Rabinovich E, et al. Relationship of DNA methylation and gene expression in idiopathic pulmonary fibrosis. Am J Respir Crit Care Med 2014; 190: 1263-1272.

Dorr KM, Amin NM, Kuchenbrod LM, et al. Caszl is required for cardiomyocyte G1-to-S phase progression during mammalian cardiac development. Development 2015; 142: 2037-2047.

Charpentier MS, Taylor JM, Conlon FL. The CASZ1/Egfl7 transcriptional pathway is required for RhoA expression in vascular endothelial cells. Small GTPases 2013; 4: 231-235.

Liu Z, Naranjo A, Thiele CJ. CASZ1b, the short isoform of CASZ1 gene, coexpresses with CASZ1a during neurogenesis and suppresses neuroblastoma cell growth. PLoS One 2011; 6: e18557.

Marques-Rocha JL, Samblas M, Milagro FI, et al. Noncoding RNAs, cytokines, and inflammation-related diseases. FASEB J 2015; 29: 3595-3611.

Jung HJ, Suh Y. MicroRNA in aging: from discovery to biology. Curr Genomics 2012; 13: 548-557.

Dimmeler S, Nicotera P. MicroRNAs in age-related diseases. EMBO Mol Med 2013; 5: 180-190.

Gorospe M, Abdelmohsen K. MicroRegulators come of age in senescence. Trends Genet 2011; 27: 233-241.

Pandit KV, Milosevic J. MicroRNA regulatory networks in idiopathic pulmonary fibrosis. Biochem Cell Biol 2015; 93: 129-137.

$$
\text { 191-199. }
$$

Milosevic J, Pandit K, Magister M, et al. Profibrotic role of miR-154 in pulmonary fibrosis. Am J Respir Cell Mol Biol 2012; 47: 879-887.

Berschneider B, Ellwanger DC, Baarsma HA, et al. miR-92a regulates TGF- $\beta 1$-induced WISP1 expression in pulmonary fibrosis. Int J Biochem Cell Biol 2014; 53: 432-441.

Idiopathic Pulmonary Fibrosis Clinical Research Network, Raghu G, Anstrom KJ, et al. Prednisone, azathioprine, and N-acetylcysteine for pulmonary fibrosis. N Engl J Med 2012; 366: 1968-1977.

Richeldi L, du Bois RM, Raghu G, et al. Efficacy and safety of nintedanib in idiopathic pulmonary fibrosis. N Engl J Med 2014; 370: 2071-2082.

King TE, Bradford WZ, Castro-Bernardini S, et al. A phase 3 trial of pirfenidone in patients with idiopathic pulmonary fibrosis. N Engl J Med 2014; 370: 2083-2092.

Puglisi S, Torrisi SE, Vindigni V, et al. New perspectives on management of idiopathic pulmonary fibrosis Ther Adv Chronic Dis 2016; 7: 108-120.

Henderson WR Jr, Chi EY, Ye X, et al. Inhibition of Wnt/beta-catenin/CREB binding protein (CBP) signaling reverses pulmonary fibrosis. Proc Natl Acad Sci USA 2010; 107: 14309-14314.

Wang $\mathrm{C}$, Zhu $\mathrm{H}$, Sun Z, et al. Inhibition of Wnt/ $\beta$-catenin signaling promotes epithelial differentiation of mesenchymal stem cells and repairs bleomycin-induced lung injury. Am J Physiol Cell Physiol 2014; 307: C234-C244.

Wang C, Dai J, Sun Z, et al. Targeted inhibition of disheveled PDZ domain via NSC668036 depresses fibrotic process. Exp Cell Res 2015; 331: 115-122.

Lauth M, Bergström A, Shimokawa T, et al. Inhibition of GLI-mediated transcription and tumor cell growth by small-molecule antagonists. Proc Natl Acad Sci USA 2007; 104: 8455-8460.

Moshai EF, Wémeau-Stervinou L, Cigna N, et al. Targeting the hedgehog-glioma-associated oncogene homolog pathway inhibits bleomycin-induced lung fibrosis in mice. Am J Respir Cell Mol Biol 2014; 51: 11-25. 
ClinicalTrials.gov. A study of vismodegib (GDC-0449, hedgehog pathway inhibitor) with concurrent chemotherapy and bevacizumab as first-line therapy for metastatic colorectal cancer. https://clinicaltrials.gov/ct2/ show/NCT00636610 Date last updated: June 1, 2012.

ClinicalTrials.gov. Hedgehog inhibition for pancreatic ductal adenocarcinoma (PDAC) in the preoperative setting (HIPPoS). https://clinicaltrials.gov/ct2/show/NCT01096732 Date last updated: January 7, 2014.

ClinicalTrials.gov. A study of hedgehog pathway inhibitor GDC-0449 in patients with locally advanced or metastatic solid tumors that are refractory to standard therapy or for whom no standard therapy exists. https:// clinicaltrials.gov/ct2/show/NCT00968981 Date last updated: November 3, 2015.

Liu G, Friggeri A, Yang Y, et al. miR-21 mediates fibrogenic activation of pulmonary fibroblasts and lung fibrosis. J Exp Med 2010; 207: 1589-1597.

Yang S, Banerjee S, de Freitas A, et al. Participation of miR-200 in pulmonary fibrosis. Am J Pathol 2012; 180: 484-493.

Xiao J, Meng XM, Huang XR, et al. miR-29 inhibits bleomycin-induced pulmonary fibrosis in mice. Mol Ther 2012; 20: 1251-1260.

Montgomery RL, Yu G, Latimer PA, et al. MicroRNA mimicry blocks pulmonary fibrosis. EMBO Mol Med 2014; 6: 1347-1356.

Das S, Kumar M, Negi V, et al. MicroRNA-326 regulates profibrotic functions of transforming growth factor- $\beta$ in pulmonary fibrosis. Am J Respir Cell Mol Biol 2014; 50: 882-892. van der Ree MH, van der Meer AJ, de Bruijne J, et al. Long-term safety and efficacy of microRNA-targeted therapy in chronic hepatitis C patients. Antiviral Res 2014; 111: 53-59.

Haefliger B, Prochazka L, Angelici B, et al. Precision multidimensional assay for high-throughput microRNA drug discovery. Nat Commun 2016; 7: 10709.

Lv XX, Wang XX, Li K, et al. Rupatadine protects against pulmonary fibrosis by attenuating PAF-mediated senescence in rodents. PLoS One 2013; 8: e68631.

Iglesias-Bartolome R, Patel V, Cotrim A, et al. mTOR inhibition prevents epithelial stem cell senescence and protects from radiation-induced mucositis. Cell Stem Cell 2012; 11: 401-414.

McCormack FX, Inoue Y, Moss J, et al. Efficacy and safety of sirolimus in lymphangioleiomyomatosis. $\mathrm{N} \mathrm{Engl} \mathrm{J}$ Med 2011; 364: 1595-1606.

ClinicalTrials.gov. Double-blind placebo-controlled pilot study of sirolimus in idiopathic pulmonary fibrosis (IPF). https://clinicaltrials.gov/ct2/show/NCT01462006 Date last updated: May 12, 2016.

Gorin Y, Cavaglieri RC, Khazim K, et al. Targeting NADPH oxidase with a novel dual Nox1/Nox4 inhibitor attenuates renal pathology in type 1 diabetes. Am J Physiol Renal Physiol 2015; 308: F1276-F1287.

Deliyanti D, Wilkinson-Berka JL. Inhibition of NOX1/4 with GKT137831: a potential novel treatment to attenuate neuroglial cell inflammation in the retina. J Neuroinflammation 2015; 12 : 136.

Zhao QD, Viswanadhapalli S, Williams P, et al. NADPH oxidase 4 induces cardiac fibrosis and hypertrophy through activating Akt/mTOR and NFKB signaling pathways. Circulation 2015; 131: 643-655.

Idiopathic Pulmonary Fibrosis Clinical Research Network, Martinez FJ, de Andrade JA, et al. Randomized trial of acetylcysteine in idiopathic pulmonary fibrosis. N Engl J Med 2014; 370: 2093-2101.

Zhu Y, Tchkonia T, Pirtskhalava T, et al. The Achilles' heel of senescent cells: from transcriptome to senolytic drugs. Aging Cell 2015; 14: 644-658.

Chang J, Wang Y, Shao L, et al. Clearance of senescent cells by ABT263 rejuvenates aged hematopoietic stem cells in mice. Nat Med 2016; 22: 78-83.

Soria-Valles C, Osorio FG, Gutiérrez-Fernández A, et al. NF- $\kappa$ B activation impairs somatic cell reprogramming in ageing. Nat Cell Biol 2015; 17: 1004-1013.

Soria-Valles C, Osorio FG, López-Otín C. Reprogramming aging through DOT1L inhibition. Cell Cycle 2015; 14: 3345-3346. 